\title{
Spatial distribution and magnetism in poly-Cr-doped GaN from first principles
}

\author{
X. Y. Cui, ${ }^{1}$ J. E. Medvedeva, ${ }^{2}$ B. Delley, ${ }^{3}$ A. J. Freeman, ${ }^{4}$ and C. Stampfl ${ }^{1}$ \\ ${ }^{1}$ School of Physics, The University of Sydney, Sydney 2006, New South Wales, Australia \\ ${ }^{2}$ Department of Physics, University of Missouri, Rolla, Missouri 65409, USA \\ ${ }^{3}$ Paul Scherrer Institut WHGA/123 CH-5232 Villigen PSI, Switzerland \\ ${ }^{4}$ Department of Physics and Astronomy, Northwestern University, Evanston, Illinois 60208-3112, USA
}

(Received 1 December 2006; published 20 April 2007)

\begin{abstract}
Large scale density-functional theory calculations have been performed to understand the spatial distribution and magnetic coupling of $\mathrm{Cr}$-doped $\mathrm{GaN}$, in which exhaustive structural and magnetic configurations have been investigated by doping of up to five $\mathrm{Cr}$ atoms in large supercells. Our results provide direct evidence that the distribution of the doped magnetic ions is neither homogeneous nor random as widely assumed previously. Rather, under both Ga-rich and N-rich growth conditions, the $\mathrm{Cr}$ atoms have a strong tendency to form substitutional, embedded clusters with short-range magnetic interactions maintaining the wurtzite structure. Significantly, while the ferromagnetic state is favored for pair doping, for more than two-Cr-atom clustering configurations, states containing antiferromagnetic or ferrimagnetic coupling with net spins in the range of $0.06-1.47 \mu_{B} / \mathrm{Cr}$ are preferred. The formation of embedded clusters leads to notable local structural distortions and considerable magnetic moments on the Cr-bonded $\mathrm{N}$ atoms. Also importantly, the electrical properties (metallic, half-metallic, or semiconducting) are found to strongly depend on the dopant concentration. We propose a picture where various cluster configurations coexist and the statistical distribution and associated magnetism depend sensitively on sample growth details. The results obtained are in agreement with recent experiments. Such a view can explain many hitherto puzzling experimental observations, e.g., the much lower value of the measured mean saturation magnetic moment on $\mathrm{Cr}$ as compared to the theoretically predicted value for the isolated dopants; the anomalous lattice constant change in relation to the dopant concentration and temperature; and the strong dependence of the magnetization on the $\mathrm{Cr}$ concentration, growth temperature, and annealing. We find a similar behavior for $\mathrm{Mn}$ in $\mathrm{GaN}$ and $\mathrm{Cr}$ and $\mathrm{Mn}$ in $\mathrm{AlN}$ and argue that such a scenario may also hold for other dilute magnetic semiconductor systems.
\end{abstract}

DOI: 10.1103/PhysRevB.75.155205 PACS number(s): 75.50.-y, 71.23.-k, 71.55.Eq, 85.75. $-\mathrm{d}$

\section{INTRODUCTION}

Since the discovery of carrier-induced ferromagnetism in InMnAs ${ }^{1}$ and GaMnAs, ${ }^{2}$ III-V type dilute magnetic semiconductors (DMSs) have attracted intense attention as they offer the potential to integrate magnetism with well-established semiconductor based technologies, which could lead to a new generation of multifunctional spintronics devices. ${ }^{3,4}$ Now, GaN is a well-known wide bandgap $(3.39 \mathrm{eV})$ semiconductor having the wurtzite structure. Compared to the much studied Mn:GaAs system, where the Curie temperature is about $170 \mathrm{~K}, \mathrm{Cr}: \mathrm{GaN}$-based systems are expected to be one of the promising candidates for room temperature DMS due to a number of appealing properties: (i) predicted longer electron spin lifetimes and distances due to the reduced spinorbit coupling, ${ }^{5,6}$ (ii) fewer compensating defects, ${ }^{7}$ (iii) higher predicted $^{8}$ and experimentally confirmed ${ }^{9-12}$ Curie temperatures $\left(T_{c}\right)$, up to $900 \mathrm{~K}$, and (iv) higher conductivity than other transition metal (TM) doped GaN systems. ${ }^{13}$ In addition, since $\mathrm{Cr}$ has a larger sticking coefficient and lower vapor pressure than $\mathrm{Mn}$, it can be incorporated at higher growth temperatures. Moreover, Graf et al. ${ }^{14}$ argued that $\mathrm{Cr}-$ :GaN may simultaneously fulfill the two main prerequisites for promising spintronic materials, namely, a large concentration of localized spin and a high density of free holes. Zhou et al. ${ }^{15}$ demonstrated that Cr:GaN magnetization data exhibited no paramagnetic component at low temperature, which is superior to Mn:GaN. By combining band structure calculations with a Heisenberg model to describe magnetic properties, Sato et al. ${ }^{16}$ predicted that $\mathrm{Cr}$ substitution should lead to higher ferromagnetic $T_{c}$ than the Mn-doped III-V systems. Recently, we predicted a possibility of efficient spin injection from a ferromagnetic $\mathrm{GaN}$ :Cr electrode through an AlN tunnel barrier in Cr-doped $\mathrm{AlN} / \mathrm{GaN}(0001)$ heterostructures. ${ }^{17}$

Despite considerable efforts aimed at elucidating the origin of the ferromagnetism in TM-doped DMS, the mechanism and its universality are still under active debate. ${ }^{18,19}$ Indeed, there are many unresolved fundamental questions in this field. In particular, one striking feature is that while the magnetization indicates ferromagneticlike ordering, the observed saturation moments are normally much lower than theoretical predictions. For example, for $\mathrm{Cr}: \mathrm{GaN}$, while the predicted value is $3 \mu_{B} / \mathrm{Cr}$ from "single Cr-doped" (one atom per supercell) density-functional theory (DFT) calculations, the experimentally observed values are normally much smaller, ranging from 0.45 to $1.8 \mu_{B} / \mathrm{Cr}{ }^{10,12,20}$ One exception is observed by Kim et al. ${ }^{21}$ in which the total effective magnetic moment is $3.17 \mu_{B} / \mathrm{Cr}$ at the $1.3 \%$ concentration. However, as the concentration increases, the mean moment significantly decreases to $1.05 \mu_{B} / \mathrm{Cr}$ and $0.79 \mu_{B} / \mathrm{Cr}$ at the concentration of 6.3 and $10.1 \%$, respectively. Also, these values seem to depend sensitively on the growth conditions, such as gas flux ratio and annealing treatment. Furthermore, previous theoretical predictions are that the system is half metallic, ${ }^{17,22,23}$ while recent experimental results show that 
$\mathrm{Cr}: \mathrm{GaN}$ is in fact semiconducting. ${ }^{20,24}$ Such a situation of course signals the complex nature of the magnetism in these materials. But the mechanism for these behaviors, particularly from the atomic scale, still remains an open question.

Experimentally, given the level of dilution of the magnetic ions (typically a few percent), it is very difficult to categorically determine the spatial distribution of the dopants and the nature of the ferromagnetism. It was argued that the low magnetic moment could be due to the presence of secondary phases or precipitates. ${ }^{25}$ However, although it is in principle difficult to preclude their presence during sample preparation, there appears to be a growing consensus that in Cr-doped GaN and AlN, secondary phases such as $\mathrm{CrN}$ [known to be antiferromagnetic (AFM) with a Neel temperature of $273 \mathrm{~K}$ (Ref. 26) or $283 \mathrm{~K}$ (Ref. 27)], $\mathrm{CrGa}_{4}$ (known to be Pauli paramagnetic with a relatively small susceptibility ${ }^{28}$ ), and $\mathrm{Cr}_{2} \mathrm{~N}$ [which might be $\mathrm{FM}$ in nature, but shows no FM signal between 85 and $500 \mathrm{~K}$ (Ref. 29)] are not responsible for the observed high $T_{c} .{ }^{9,12,20,24,30}$ Furthermore, experimental results showed that in high quality epitaxial Cr-doped III-N thin films, there are no detectable secondary phases (or that the amount of secondary phases is insignificant) and the $\mathrm{Cr}$ atoms are on a substitutional $\mathrm{Ga}$ site. $^{7,11,12,31}$ Thus the experimentally observed ferromagnetism is expected to arise from intrinsic $\mathrm{Cr}: \mathrm{GaN}$ and Cr:AlN.

From the theoretical side, one approach widely employed to study DMS is based on mean field theory (MFT), which implicitly assumes that the DMS is a more-or-less random alloy and the spin-spin coupling is assumed to be a longrange interaction. Using MFT, it was predicted that structural disorder enhances the ferromagnetic (FM) transition temperature in $\mathrm{Mn}: \mathrm{GaAs},{ }^{32}$ and further, reported that a correlated defect distribution (involving $\mathrm{Mn}$ and As-antisite clustering) is important. ${ }^{33}$ In contrast, $\mathrm{Xu}$ et al. ${ }^{34}$, in studying $\mathrm{Cr}$ and $\mathrm{Mn}$ in $\mathrm{GaAs}$ and $\mathrm{GaN}$, and taking into account the magnetic atom pair interaction, concluded that disorder (and clustering) reduces the critical temperature, while ordering of the dopants increases it. Now, in general, MFT can only describe the averaged electronic properties, and local information such as the magnetic structure and magnetic coupling are not obtainable. Moreover, other recent studies have reported the relevance of a random distribution of magnetic impurities for the $T_{c}$, in particular, for $\mathrm{Mn}: \mathrm{GaN}$ in which the magnetic interactions are short ranged. ${ }^{35-37}$

First-principles DFT calculations have also been performed for these systems. In principle, this allows one to treat not only random disorder but also dopants with correlated positions. To date, the majority of such investigations in DMS, and Cr:GaN, Mn:GaN, and Mn:GaAs systems in particular, have focused on "single" 38 atom doping on the cation sites, where changes in the concentration is achieved by varying the size of the supercells. This clearly results in a homogeneous distribution of the TM atoms where the effects of disorder and other ordering configurations are neglected. Furthermore, within this scheme, FM coupling is assumed between the ions in the periodic neighboring supercells. There have been several studies addressing the effects of structural disorder in DMS: Recently, several groups investigated the tendency of clustering and magnetic coupling by performing "pair doping" (two dopant TM atoms in a supercell) $22,39-43$ and concluded that the FM state is favorable over the AFM state. The calculated magnetic moments are much higher than experimental observations, as mentioned above.

The consideration of clustering of the magnetic dopants in DMS arises mainly due to the seminal work of van Schilfgaarde and Mryasov, ${ }^{44}$ which was the first theoretical work to suggest that the magnetic impurities in TM-doped III-V semiconductors may aggregate into small nanoclusters of a few magnetic atoms. Subsequently, Mahadevan and Zunger ${ }^{45}$ found that the ferromagnetism in Mn:GaAs arises from isolated Mn atoms as well as from strongly Coulomb-stabilized substitutional-interstitial complexes. Drchal et al. ${ }^{46}$ examined the stability of these alloys with respect to segregation and estimated the formation energies of antisite defects and substitutional and interstitial $\mathrm{Mn}$ atoms in GaAs by treating magnetic disorder within the disordered local moment model. They found a strong tendency of substitutional Mn atoms in Mn:GaAs to the aggregate with the parallel magnetic alignment. Moreover, Raebiger et $a l^{47}$ investigated purely substitutional Mn clusters in Mn:GaAs and reported that the most energetically favorable clusters consist of $\mathrm{Mn}$ atoms surrounding a one center As atom.

Conceptually, it is convenient to consider the nature of the magnetic coupling by investigating the behavior of pairs of atoms, namely, by comparing the stability of the FM and AFM states and determining which is the ground state. However, this "pair model" may be invalid or incomplete, particularly in dilute doped systems, since the effect of a third (or more) TM dopants may spoil this simplistic description. Recently, we briefly reported an extensive DFT investigation of the distribution of the doped ions and the magnetic properties of $\mathrm{Cr}: \mathrm{GaN}$ by the doping of up to $5 \mathrm{Cr}$ atoms in large supercells. Indeed, we found that the stability and magnetic properties are fundamentally altered by the presence of the additional $\mathrm{Cr}$ atoms, demonstrating that such "poly-doping" (in contrast to single and pair doping) is crucial to give a correct description regarding the magnetic interactions. While for pair doping, the most favorable configuration is FM, in agreement with previous calculations, ${ }^{22}$ for more than two-Cr-atom doping, states containing AFM coupling with net spin in the range of $0.06-1.47 \mu_{B} / \mathrm{Cr}$ are found to be stable. The increased doping concentration and temperature should enhance the clustering behavior and consequently decrease the effective magnetic moment. Thus, we conclude that the spatial distribution of the doped magnetic ions is neither random nor homogeneous: the $\mathrm{Cr}$ atoms prefer to form "embedded clusters" and as the cluster size grows, the states containing AFM couplings with net spin moment close to the experimental values are favored.

Clearly, only when one has knowledge of the true atomic geometry and composition can one hope to obtain an understanding of the associated structural, electronic, and magnetic properties of these materials. In this paper, we present a detailed description of a comprehensive investigation of various doping concentrations and configurations in large supercells of $\mathrm{Cr}: \mathrm{GaN}$, including substitutional single, pair, and poly-Cr configurations, substitutional-interstitial complexes, $\mathrm{Cr}-\mathrm{Cr}$ bonded configurations, $\mathrm{Cr}-V_{\mathrm{N}}(\mathrm{Cr}$ with $\mathrm{N}$ vacancy) 
TABLE I. Calculated and experimental lattice constants for bulk $\mathrm{Ga}, \mathrm{Cr}$, and $\mathrm{Cr}_{2} \mathrm{~N}$.

\begin{tabular}{lccc}
\hline \hline & & \multicolumn{2}{c}{ Lattice constants $(\AA)$} \\
\cline { 3 - 4 } & Space group & Present & Experimental \\
\hline $\mathrm{Cr}$ & $\operatorname{Im} \overline{3} m$ & $a=2.854$ & $a=2.88$ (Ref. 65) \\
$\mathrm{Ga}$ & $C m c a$ & $a=4.640, b=7.798, c=4.529$ & $a=4.638, b=7.816, c=4.541$ (Ref. 66) \\
$\mathrm{Cr}_{2} \mathrm{~N}$ & $P 63 / m m c$ & $a=2.748, c=4.429$ & $a=2.832, c=4.475$ (Ref. 61) \\
\hline \hline
\end{tabular}

complexes and cluster-cluster interactions. We use a unified quantity, the formation energy, to evaluate their relative stability. Furthermore, to study the influence of the growth conditions, we calculated the formation energy under $\mathrm{N}$-rich and Ga-rich conditions. Also we address the importance of the size of the supercell (correlated to the doping concentration) and the necessity of atomic relaxation in DMS cluster-doping calculations, which was neglected in some previous polydoping investigations. ${ }^{47,48}$ The paper is organized as follows. In Sec. II the calculation method and computational details are described and in Sec. III, the results are presented. These include single- and poly-doping substitutional configurations, complexes involving interstitial-substitutional $\mathrm{Cr}$, as well as structures involving $\mathrm{Cr}-\mathrm{Cr}$ bonding. In $\mathrm{Sec}$. IV we discuss the feasibility of clustering and propose a model regarding the spatial distribution and magnetism in $\mathrm{Cr}: \mathrm{GaN}$ which helps to understand some puzzling experimental observations. We summarize our main conclusions in Sec. V.

\section{CALCULATION DETAILS}

The DFT calculations are performed using the periodic DMol $^{3} \operatorname{code}^{49}$ with the generalized gradient approximation ${ }^{50}$ (GGA) for the exchange-correlation functional and with density-functional semilocal pseudopotentials (DSPP). ${ }^{51}$ In this scheme, all electrons for $\mathrm{N}$ are included, while for $\mathrm{Ga}$ and $\mathrm{Cr}$ the $3 d^{10} 4 s^{2} 4 p^{1}$ and $3 s^{2} 3 p^{6} 3 d^{5} 4 s^{1}$ electrons are treated as valence electrons, respectively. A double set of numerical valence functions with a local basis cutoff of 11.0 a.u. is used. The optimized wurtzite $\mathrm{GaN}$ lattice constants are $a$ $=3.184 \AA$ and $c=5.184 \AA$ with internal parameter $u$ $=0.3765$, which are in good agreement with experimental and previous theoretical results. ${ }^{52}$ Full atomic relaxation is included in all calculations. Various 32-atom $(2 a \times 2 a \times 2 c)$, 72-atom $(3 a \times 3 a \times 2 c)$, 96-atom (cubic) $(2 \sqrt{3} a \times 3 a \times 2 c)$, 108-atom $(3 a \times 3 a \times 3 c), \quad 128$-atom $(4 a \times 4 a \times 2 c)$, 256atom $(4 a \times 4 a \times 4 c)$, and 300 -atom $(5 a \times 5 a \times 3 c)$ supercells are used. In the hexagonal supercells, the separation between dopants in neighboring supercells can be quite different along different directions. The cubic 96-atom-cell avoids this problem by having translation vectors that are mutually perpendicular, leading to a cell with orthorhombic symmetry. Large supercells ensure that for each configuration studied, the interaction between neighboring supercells is negligible, and so the results presented are for truly isolated configurations. Convergence as a function of supercell size has been checked for all the favorable structures in this paper. We find that the size of the supercells is crucial in order to determine the correct magnetic coupling and electronic structure. Reciprocal space Monkhorst-Pack ${ }^{53} \mathbf{k}$-point meshes of $8 \times 8$ $\times 6$ for the 32 -atom, $4 \times 4 \times 3$ for the 72 -atom; $2 \times 2 \times 2$ for the 96-, 108-, 128-atom; $2 \times 2 \times 1$ for 256-atom; and $1 \times 1$ $\times 2$ for the 300 -atom supercells are employed. The accuracy of the present $\mathrm{DMol}^{3}$ calculations is demonstrated by the good agreement with those using the highly accurate full-potential linearized-augmented plane-wave (FLAPW) code for the $\mathrm{Cr}$ :AlN/GaN interfaces. ${ }^{17}$ Moreover, the $\mathrm{DMol}^{3}$ code has been employed to study the magnetism in gas-phase $\mathrm{Cr}$ clusters $^{54}$ and $\mathrm{V}$ - and Cr-doped BeTe systems. ${ }^{55}$

To evaluate the relative stability of various configurations, the formation energy is calculated as

$$
E^{f}=E_{\mathrm{Cr}: \mathrm{GaN}}-E_{\mathrm{ref} \mathrm{GaN}}-n \mu_{\mathrm{Cr}}+m \mu_{\mathrm{Ga}}+l \mu_{\mathrm{N}},
$$

where $E_{\mathrm{Cr}: \mathrm{GaN}}$ and $E_{\text {ref GaN }}$ are the total energies of Cr-doped $\mathrm{GaN}$ and the pure $\mathrm{GaN}$ reference structure (as calculated with the same size supercell), respectively. $\mu_{\mathrm{Ga}}, \mu_{\mathrm{Cr}}$, and $\mu_{\mathrm{N}}$ are the atom chemical potentials of $\mathrm{Ga}, \mathrm{Cr}$, and $\mathrm{N}$, while the integers $n, m$, and $l$ are the number of doped $\mathrm{Cr}$ atoms, and substituted $\mathrm{Ga}$ and $\mathrm{N}$ atoms, respectively. The chemical potentials depend on the experimental conditions under which the material is grown. In order to determine these quantities, we invoke the relationship $\mu_{\mathrm{Ga}}+\mu_{\mathrm{N}}=\mu_{\mathrm{GaN}}$, assuming both species are in thermal equilibrium with GaN. Furthermore, the chemical potentials must satisfy the boundary conditions $\mu_{\mathrm{N}}<1 / 2 \mu_{\mathrm{N}_{2}}$ and $\mu_{\mathrm{Ga}}<\mu_{\mathrm{Ga} \text { (bulk) }}$ (if this were not the case, then $\mathrm{GaN}$ would be thermodynamically unstable with respect to the formation of $\mathrm{N}_{2}$ molecules or bulk $\mathrm{Ga}$ ). When imposing certain growth conditions [nitrogen-rich $\left(\mu_{\mathrm{N}}=1 / 2 \mu_{\mathrm{N}_{2}}\right)$ or gallium-rich $\left(\mu_{\mathrm{Ga}}=\mu_{\mathrm{Ga} \text { (bulk) }}\right)$ conditions], the chemical potential for the other species can be determined from the above (thermal equilibrium) relationship. The atomic chemical potential for $\mathrm{Cr}$ is assumed to be determined by equilibrium with bulk $\mathrm{Cr}_{2} \mathrm{~N}$, which is the most stable nitride of chromium. Another motivation for using $\mathrm{Cr}_{2} \mathrm{~N}$ is that under nonoptimized growth conditions, second phases of $\mathrm{Cr}_{2} \mathrm{~N}$ are detected in $\mathrm{AlN}$ in experiments. ${ }^{56,57}$ Under nitrogen-rich conditions, $\quad \mu_{\mathrm{Cr}}=1 / 2\left[E_{\mathrm{Cr}_{2} \mathrm{~N} \text { (bulk) }}-1 / 2 E_{\mathrm{N}_{2}}\right]$ and $\mu_{\mathrm{Ga}}$ $=E_{\mathrm{GaN}(\text { bulk })}-1 / 2 E_{\mathrm{N}_{2}}$; and under gallium rich conditions, $\mu_{\mathrm{N}}$ $=E_{\mathrm{GaN}(\text { bulk })}-E_{\mathrm{Ga} \text { (bulk) }}$ and $\mu_{\mathrm{Cr}}=1 / 2\left[E_{\mathrm{Cr}_{2} \mathrm{~N} \text { (bulk) }}-\left(E_{\mathrm{GaN} \text { (bulk) }}\right.\right.$ $\left.\left.-E_{\mathrm{Ga} \text { (bulk) }}\right)\right]$. Here $E_{\mathrm{Cr}_{2} \mathrm{~N} \text { (bulk) }}, E_{N_{2}}, E_{\mathrm{GaN} \text { (bulk) }}$ and $E_{\mathrm{GaN} \text { (bulk) }}$, are the total energies of bulk $\mathrm{Cr}_{2} \mathrm{~N}$, the nitrogen molecule, bulk $\mathrm{GaN}$, and bulk Ga, respectively. The calculated structural parameters for bulk $\mathrm{Cr}, \mathrm{Ga}$, and $\mathrm{Cr}_{2} \mathrm{~N}$ are summarized in Table I and compared with experimental data. For bulk $\mathrm{Cr}$, we used the body-centered-cubic (bcc) structure in nonmag- 
netic state. We are aware that the ground magnetic state for bulk $\mathrm{Cr}$ is in fact a long-period spin-density wave. ${ }^{58}$ However, the energy difference between the nonmagnetic phase and ground state is only $1.4 \mathrm{meV} / \mathrm{Cr},{ }^{60}$ or $7-23 \mathrm{meV} / \mathrm{Cr}$ as a function of wave vector, ${ }^{58}$ and thus can be neglected in the present study. The calculated heat of formation of $\mathrm{GaN}$ is $-1.04 \mathrm{eV}$, which is in good agreement with the established data (experimental value is $-1.17 \mathrm{eV}$ ) ${ }^{59}$ For bulk $\mathrm{Cr}_{2} \mathrm{~N}$, our spin-polarized DFT calculations show it is nonmagnetic and the calculated heat of formation is $-0.93 \mathrm{eV}$ per formula unit with the $P 63 / m m c$ symmetry ${ }^{61}$ which is close to the experimental value of $-1.01 \mathrm{eV}^{62}$ For the $\mathrm{N}_{2}$ molecule, the calculated binding energy, bond length and vibrational frequency are $9.98 \mathrm{eV}, 1.123 \AA$, and $2369 \mathrm{~cm}^{-1}$, respectively, which are in good agreement with the experimental values of $9.91 \mathrm{eV}, 1.098 \AA$, and $2360 \mathrm{~cm}^{-1}$, respectively, and with other theoretical results. ${ }^{52}$

\section{RESULTS}

In general, one of the most interesting and challenging aspects in DMS is to determine the arrangement of, and the subtle interplay between the magnetic dopants. We first determine the most favorable site for the case of "single" and "pair" Cr doping, i.e., one and two $\mathrm{Cr}$ atoms in the supercell. Then, we explore the possibility of direct $\mathrm{Cr}-\mathrm{Cr}$ bonding configurations as well as the interaction of $\mathrm{Cr}$ with a $\mathrm{N}$ vacancy. Subsequently, we investigate "poly- $\mathrm{Cr}_{\mathrm{Ga}}$ doping" involving pure substitutional configurations as well as substitutionalinterstitial complexes. As will be described below, the energetically favorable configurations are depicted in Fig. 1 and the associated spin moments, formation energies and magnetic ground states are listed in Table II.

\section{A. Single $\mathrm{Cr}$ doping}

For the doping of a single $\mathrm{Cr}$ atom, four high-symmetry sites, including two substitutional $\left(\mathrm{Cr}_{\mathrm{Ga}}\right.$ and $\left.\mathrm{Cr}_{\mathrm{N}}\right)$ and two interstitial sites (tetrahedral $T$ ) and (octahedral $O)\left(\mathrm{Cr}_{i-O}\right.$ and $\mathrm{Cr}_{i-T}$ ) are considered. The $T$ site corresponds to the center of the line joining the closest nonbonded $\mathrm{Ga}$ and $\mathrm{N}$ atoms along the $c$ axis. It has two nearest neighbors (one cation and one anion) and six next-nearest neighbors (three cations and three anions). The $O$ site is located at the center of the hexagonal channel, and has six nearest neighbors (three cations and three anions). As expected, and in agreement with experiments, ${ }^{12} \mathrm{Cr}_{\mathrm{Ga}}$, with integral magnetic moment of $3 \mu_{B} / \mathrm{Cr}$, is much more energetically favored than all the other three configurations, by $5-9 \mathrm{eV} / \mathrm{Cr}$ under both N-rich and Ga-rich conditions. The other substitutional site $\mathrm{Cr}_{\mathrm{N}}$ gives an atomic moment $1.07 \mu_{B} / \mathrm{Cr}$. For the interstitial doping, the $\mathrm{Cr}_{i-O}$ site is lower in energy than the $\mathrm{Cr}_{i-T}$ site by $1.59 \mathrm{eV}$ (32-atom cell) and $1.67 \mathrm{eV}$ (72-atom cell). Both $\mathrm{Cr}_{i-O}$ and $\mathrm{Cr}_{i-T}$ are predicted to be virtually nonmagnetic. The absence of magnetism of a single $\mathrm{Cr}$ at interstitial sites may be attributed to the compressed interatomic electron motion in the limited space. ${ }^{63}$

The calculated magnetic moment of $3 \mu_{B} / \mathrm{Cr}$ for $\mathrm{Cr}_{\mathrm{Ga}}$ can be understood based on the fact that when the neutral $\mathrm{Cr}$ (a)
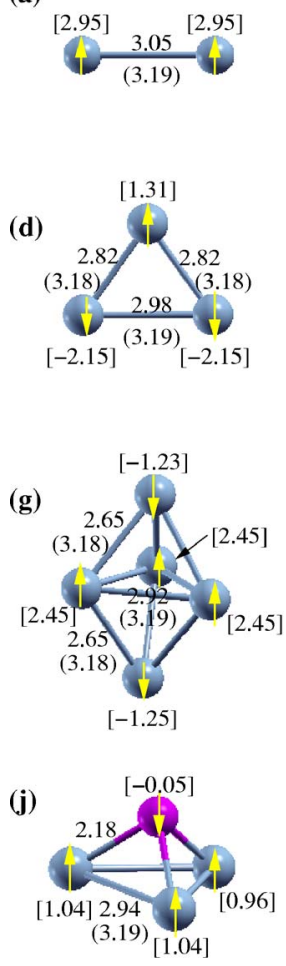

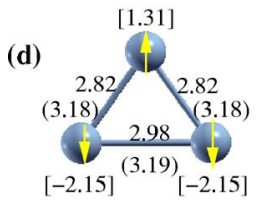

(b)

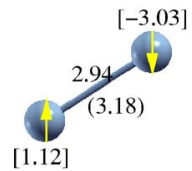

(e)

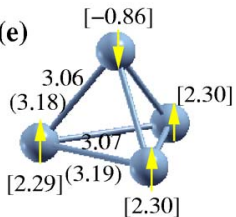

(h)
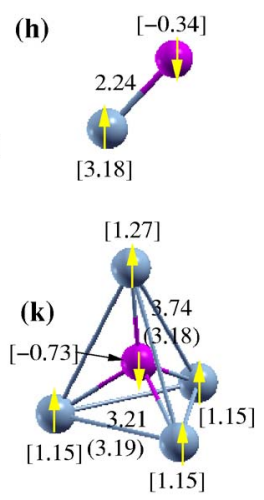

(c)
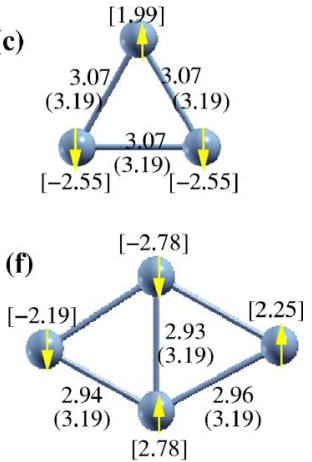

(i)

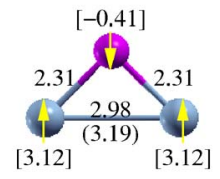

(l)

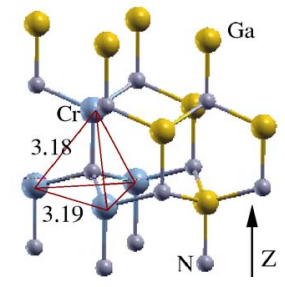

FIG. 1. (Color online) Optimized Cr clustering geometries and magnetic structures. The light (blue) circles represent substitutional $\mathrm{Cr}_{\mathrm{Ga}}$ and the dark (pink) interstitial $\mathrm{Cr}_{i-O}$ in (h)-(k). The $\mathrm{Cr}-\mathrm{Cr}$ distances before (in parenthesis) and after relaxation are in $\AA$, and the arrows indicate the directions of the local atomic spin for the magnetic ground state. The atomic spin moment values (in square brackets) are in $\mu_{B}$. Note that the links between $\mathrm{Cr}_{\mathrm{Ga}}$ atoms are only a guide to the eye since in the supercells, the $\mathrm{Cr}$ atoms are of course bonded to $\mathrm{N}$ and $\mathrm{Ga}$ atoms, which are omitted here. See Fig. 1(1), showing the actual configuration of structure 1(e) in the "real" GaN supercell.

atom with electronic configuration $3 d^{5} 4 s^{1}$ substitutes on the cation site, the effective electronic configuration is $3 d^{3} 4 s^{0}$ as three electrons will saturate the four $\mathrm{N}$ dangling bonds. In tetrahedral-coordinated III-V semiconductors, the TM $d$ states split into a triply degenerate $t_{2}$ and a low-lying doubly degenerate $e$ level for each spin due to the crystal field splitting. These states are further split by the Jahn-Teller effect. Density of states (DOS) analysis clearly shows that two $d$ electrons occupy the two $e$ orbitals with the same spin and one $d$ electron occupies the $t_{2}$ orbital (see Fig. 2). The integer magnetic moment can be well interpreted by simple crystal field theory and by considering the facts that (i) the bandgap of the host semiconductor GaN (calculated value $2.58 \mathrm{eV}$ compared to the experimental value of $3.47 \mathrm{eV}$; DFT is well known to underestimate semiconductor bandgaps ${ }^{64}$ ), serves as the "arena" for the localized $d$-band electrons and (ii) the hybridization region (located in the energy range of $-8 \mathrm{eV}$ to $-3 \mathrm{eV}$ and dominated by $\mathrm{Cr}-d$ and $\mathrm{N}-2 p$ interactions) is well below the Fermi energy. Consequently, while the hybridization does not contribute to the total magnetic moment per cell, it does, however, affect the atomic magnetic moments. 
TABLE II. Formation energies (N-rich, and Ga-rich conditions), net spin values, and ground magnetic states for the various configurations considered. The quantities in bold type are the lowest energy structures. Structures a-k are shown in Fig. 1 and structures I-IV are shown in Fig. 3 (see text).

\begin{tabular}{lccccc}
\hline \hline Structure & $\begin{array}{c}\text { No. of Cr } \\
\text { atoms }\end{array}$ & $\begin{array}{c}E_{\mathrm{N}-\text {-rich }}^{f} \\
(\mathrm{eV} / \mathrm{Cr})\end{array}$ & $\begin{array}{c}E_{\mathrm{Ga}-\text { rich }}^{f} \\
(\mathrm{eV} / \mathrm{Cr})\end{array}$ & $\begin{array}{c}\text { Net spin } \\
(\mu B / \mathrm{Cr})\end{array}$ & $\begin{array}{r}\text { Ground } \\
\text { Single-doping Configurations }\end{array}$ \\
\hline $\mathrm{Cr}_{\mathrm{Ga}}$ & 1 & $\mathbf{1 . 5 2}$ & $\mathbf{2 . 0 4}$ & $\mathbf{3}$ & \\
$\mathrm{Cr}_{\mathrm{N}}$ & 1 & 8.84 & 7.28 & 1.07 & \\
$\mathrm{Cr}_{i-\mathrm{O}}$ & 1 & 8.07 & 7.55 & 0.02 & \\
$\mathrm{Cr}_{i-T}$ & 1 & 9.74 & 9.22 & 0 & \\
$\mathrm{Cr}_{\mathrm{Ga}}+\mathrm{N}_{\text {vacancy(1) }}$ & 1 & 11.17 & 10.65 & 3.63 & \\
$\mathrm{Cr}_{\mathrm{Ga}}+\mathrm{N}_{\text {vacancy(2) }}$ & 1 & 11.22 & 10.74 & 3.61 & \\
& $\mathrm{Cr}-\mathrm{Cr}$ bonded configurations & & \\
\hline I & 2 & 5.08 & 4.56 & 0.03 & AFM \\
II & 2 & 5.24 & 4.72 & 0.04 & AFM \\
III & 2 & 4.11 & 4.11 & $\mathbf{0 . 0 0}$ & AFM \\
IV & 2 & 4.23 & 4.23 & 0.11 & AFM
\end{tabular}

Substitutional configurations

\begin{tabular}{cccccc}
\hline a & $\mathbf{2}$ & $\mathbf{1 . 1 7}$ & $\mathbf{1 . 6 9}$ & $\mathbf{2 . 9 8}$ & FM \\
$\mathrm{b}$ & 2 & 1.34 & 1.86 & 0.94 & AFM \\
$\mathrm{c}$ & $\mathbf{3}$ & $\mathbf{0 . 9 8}$ & $\mathbf{1 . 5 0}$ & $\mathbf{1 . 0 4}$ & AFM \\
$\mathrm{d}$ & 3 & 1.07 & 1.59 & 1.03 & AFM \\
$\mathrm{e}$ & $\mathbf{4}$ & $\mathbf{0 . 7 7}$ & $\mathbf{1 . 2 9}$ & $\mathbf{1 . 4 7}$ & AFM \\
$\mathrm{f}$ & 4 & 0.92 & 1.44 & 0.06 & AFM \\
$\mathrm{g}$ & $\mathbf{5}$ & $\mathbf{0 . 7 3}$ & $\mathbf{1 . 2 5}$ & $\mathbf{0 . 9 7}$ & AFM
\end{tabular}

Substitutional-interstitial complexes

\begin{tabular}{llllll}
\hline $\mathrm{h}$ & 2 & 3.87 & 3.87 & 1.31 & AFM \\
$\mathrm{i}$ & 3 & 2.48 & 2.65 & 1.72 & AFM \\
$\mathrm{j}$ & 4 & 2.16 & 2.42 & 0.78 & AFM \\
$\mathrm{k}$ & 5 & 1.73 & 2.04 & 0.76 & AFM \\
\hline
\end{tabular}

\section{B. Substitutional pair Cr doping}

For "pair" $\mathrm{Cr}_{\mathrm{Ga}}$ doping, we considered seven possible atomic configurations in the 72-atom cell. These structures, along with the relative energy difference between the nonmagnetic, FM and AFM states are compiled in Table III. For simplicity, in this work, "AFM" states also include ferrimagnetism. In each geometry, one $\mathrm{Cr}$ atom is located at the origin, while the coordinates of the other $\mathrm{Cr}$ atom are given in terms of the primitive lattice vectors in the wurtzite structure. To reduce the effect of $\mathrm{Cr}-\mathrm{Cr}$ interactions between adjacent cells, a larger 108-atom-cell is used for the $\mathrm{Cr}$ atoms located at positions $(1,1,1)$ and $(1,2,1)$ (given in terms of lattice vectors). For all the structures considered, the nonmagnetic state has significantly higher energy than the FM and AFM counterparts, while the total energies of FM and AFM are rather competitive, depending on distance and orientation. The most favorable configuration is the one where two $\mathrm{Cr}$ atoms
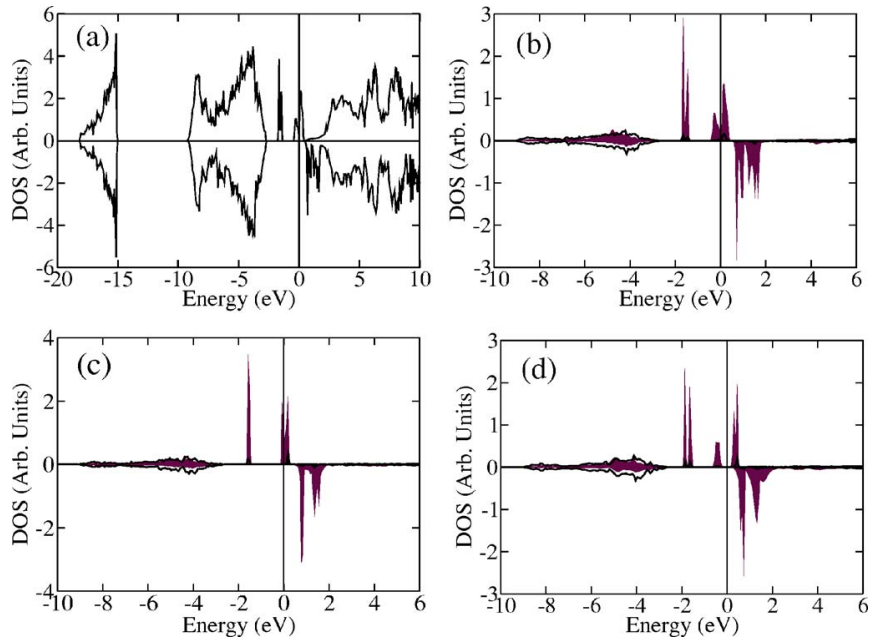

FIG. 2. (Color online) DOS for single $\mathrm{Cr}_{\mathrm{Ga}}$ in $\mathrm{Cr}: \mathrm{GaN}$ : Total DOS (a) and the projected DOS (b) as calculated in the 32-atom cell. The projected DOS calculated using a 72-atom cell (c) and in a 96-atom cell (d). In (b), (c), and (d), a smaller energy range is shown as compared to (a). The filled grey areas are for Cr-3d states and the solid lines are for the singly $\mathrm{Cr}$-bonded $\mathrm{N}$ atom $2 p$ states.

substitute the in-plane Ga atoms with a distance of $3.19 \AA$ [see Fig. 1(a)]. For this structure, while the 32-atom cell predicts the AFM state is lower in energy than the FM state by $61 \mathrm{meV}$ per cell, the 72- and 96-atom cells both show that the FM state with a moment of $2.95 \mu_{B} / \mathrm{Cr}$ is more favorable than the AFM state (with $\pm 2.50 \mu_{B} / \mathrm{Cr}$ ) by 224 and $241 \mathrm{meV}$ per cell, respectively. Thus, for this configuration at a concentration of $12.5 \%$, the AFM is predicted while at lower concentrations, 6.25 and $4.17 \%$, the FM is predicted to be the ground state. For the next most favorable structure where the two $\mathrm{Cr}$ atoms substitute nearest neighboring out-of-plane Ga sites [see Fig. 1(b)], while the 32-atom cell shows that the FM state is slightly more favorable than the AFM state by $6.8 \mathrm{meV}$, instead both the 72- and 96-atom cells predict that the AFM state (with a net spin of $0.94 \mu_{B} / \mathrm{Cr}$ ), is more stable than the FM state (with a net spin $1.95 \mu_{B} / \mathrm{Cr}$ ) by 32 and $35 \mathrm{meV}$ per cell, respectively. Note that Ref. 40 reported that $\mathrm{Cr}$-doped $\mathrm{GaN}$ is FM irrespective of $\mathrm{Cr}$ concentration. We attribute this discrepancy to the small size of the model used in Ref. 40 [a nine-layer $(1 \times 2)$ slab] since we have confirmed that the FM is predicted for the structure in Fig. 1(b) using a 16-atom $(1 \times 2 \times 2)$ cell, which has the same lateral $\mathrm{Cr}-\mathrm{Cr}$ distance. These results clearly demonstrate that for pair-doping in 32-atom (or smaller) supercells there are still considerable interactions between the magnetic dopants in the periodic neighboring supercells.

\section{C. $\mathrm{Cr}_{\mathrm{Ga}}-\mathrm{V}_{\mathrm{N}}$ complexes and direct $\mathrm{Cr}-\mathrm{Cr}$ bonded structures}

It was reported that native defects, specifically Asantisites, are important in determining the magnetic properties of Mn:GaAs, where the involvement of As-antisites results in a smaller magnetic moment. ${ }^{46,67}$ In GaN, the most common native defects are the $\mathrm{N}$ and $\mathrm{Ga}$ vacancies. ${ }^{68,69}$ Since the $\mathrm{Cr}$ atoms preferably occupy $\mathrm{Ga}$ sites, we consid- 
TABLE III. Relative total energies and atomic spin moments for the various configurations for pair doping in the 72-atom cell. One $\mathrm{Cr}$ atom is at the origin and the coordinates of the second $\mathrm{Cr}$ (II) are listed (in terms of the primitive lattice vectors). The quantities in bold type are for the ground magnetic states.

\begin{tabular}{lcccccc}
\hline \hline \multirow{2}{*}{$\begin{array}{l}\text { Coordinates } \\
\text { of Cr(II) }\end{array}$} & $\begin{array}{c}\text { Cr-Cr } \\
\text { distances }(\AA)\end{array}$ & Nonmagnetic & \multicolumn{2}{c}{ FM } & \multicolumn{2}{c}{ AFM } \\
\cline { 3 - 7 }$(1 / 3,2 / 3,1 / 2)$ & 3.180 & 0.707 & $1.08,2.82$ & 0.371 & $\mathbf{1 . 1 2 , - 3 . 0 3}$ & $\mathbf{0 . 3 3 9}$ \\
$(1,0,0)$ & 3.189 & 1.001 & $\mathbf{2 . 9 5 , 2 . 9 5}$ & $\mathbf{0}$ & $2.50,-2.46$ & 0.224 \\
$(0,0,1)$ & 5.186 & 1.296 & $\mathbf{2 . 9 5 , 2 . 9 5}$ & $\mathbf{0 . 7 4 1}$ & $2.93,-2.92$ & 0.843 \\
$(1 / 3,5 / 3,1 / 2)$ & 5.518 & 1.155 & $2.91,2.91$ & 0.627 & $\mathbf{2 . 8 6 , - 2 . 8 5}$ & $\mathbf{0 . 5 6 1}$ \\
$(4 / 3,8 / 3,0)$ & 5.524 & 1.306 & $\mathbf{2 . 9 0 , 2 . 9 0}$ & $\mathbf{0 . 8 3 2}$ & $2.93,-2.91$ & 0.851 \\
$(1,1,1)$ & 6.088 & 1.162 & $2.85,2.85$ & 0.522 & $\mathbf{2 . 8 2},-\mathbf{2 . 8 2}$ & $\mathbf{0 . 4 6 5}$ \\
$(1,2,1)$ & 7.577 & 1.356 & $\mathbf{2 . 9 3 , 2 . 9 5}$ & $\mathbf{0 . 8 2 9}$ & $2.93,-2.93$ & 0.858 \\
\hline \hline
\end{tabular}

ered the coupling between $\mathrm{Cr}_{\mathrm{Ga}}$ and neighboring $\mathrm{N}$ vacancies, as proposed in Ref. 70. In particular, two configurations are considered in the 72-atom cell: (i) the nearest-neighbor $\mathrm{N}$ (planer) vacancy and (ii) the next-nearest-neighbor $\mathrm{N}$ (apical) vacancy. Both configurations give a large magnetic moment of around $3.6 \mu_{B} / \mathrm{Cr}$. The corresponding formation energy of these complexes are 11.17 and $11.22 \mathrm{eV} / \mathrm{Cr}$ (N-rich) and 10.65 and $10.74 \mathrm{eV} / \mathrm{Cr}$ (Ga-rich), respectively (see Table II). These very high formation energies suggest that such configurations are not likely to play an important role in Cr:GaN systems.

Considering the fact that the nearest neighbor $\mathrm{Cr}-\mathrm{Cr}$ distance in bulk bcc-Cr is $2.49 \AA$, which is between the N-Ga $(1.95 \AA)$ and the Ga-Ga $(3.19 \AA)$ distances in GaN, we also investigated the possibility of direct $\mathrm{Cr}-\mathrm{Cr}$ bonded clustering, i.e., with no $\mathrm{N}$ atoms in between the two $\mathrm{Cr}$ atoms. Four structures are considered in the 72-atom cell, which are depicted in Fig. 3. Both structures I and II contain an adjacent $\mathrm{Cr}_{\mathrm{Ga}}+\mathrm{Cr}_{\mathrm{N}}$ complex, where the $\mathrm{Cr}$-atom pairs are parallel and perpendicular to the (0001) plane for structures I and II, respectively. Structures III and IV involve two neighboring $\mathrm{Cr}_{\mathrm{Ga}}$ atoms with a $\mathrm{N}$ vacancy in between. The formation energies under metal rich growth condition (i.e., the atom removed from (added to) the $\mathrm{GaN}$ is incorporated into
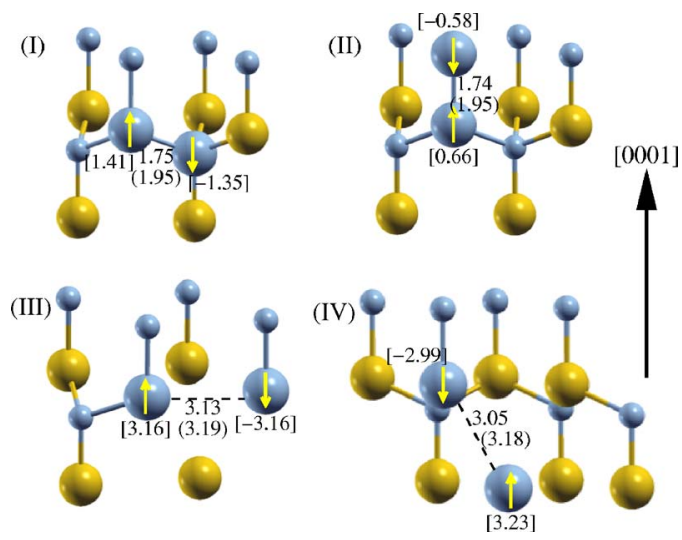

FIG. 3. (Color online) Configurations considered involving direct $\mathrm{Cr}$ - $\mathrm{Cr}$ interactions. The large light (grey) circles with arrows represent $\mathrm{Cr}$ atoms, the medium (yellow) $\mathrm{Ga}$ atoms, and the small (light grey), $\mathrm{N}$ atoms. Other notations are as in Fig. 1. (comes from) the corresponding bulk reservoir) and magnetic moments for the various magnetic states are listed in Table IV. For all these configurations, AFM states are found to be favorable over the FM state. For structures I and II shown in Figs. 3, the initial FM configurations are not stable and they merged into AFM solutions, while for structures III and IV shown in Figs. 3, the AFM is more stable than the FM state by 35 and $123 \mathrm{meV}$, respectively. The most favorable configuration is III shown in Fig. 3, where a $\mathrm{N}$ vacancy is present. Overall, the formation energies are much higher than for the two isolated $\mathrm{Cr}_{\mathrm{Ga}}$ ions. Thus, we conclude that all these configurations are unlikely to play an important role for this system. This is in agreement with recent experimental results indicating that $\mathrm{Cr}-\mathrm{Cr}$ bonding does not exist in $\mathrm{Cr}: \mathrm{GaN}^{24}$ We note that in this experimental paper, the term "clustering" refers to direct $\mathrm{Cr}-\mathrm{Cr}$ bonded structures or the formation of cubic $\mathrm{CrN}$, which fundamentally differs from the "embedded $\mathrm{Cr}-\mathrm{N}-\mathrm{Cr}$ clustering" described in the present paper.

\section{Substitutional poly-Cr doping}

Poly-doping investigations on DMSs are intrinsically complicated due to the large number of possible atomic and magnetic configurations involved. Furthermore, a polydoping study generally requires large supercells to ensure that the interaction between the neighboring supercells is negligible. For doping of up to $5 \mathrm{Cr}$ atoms in large supercells, we performed an exhaustive search for the geometric and magnetic ground state structures. For a given structure containing $n \mathrm{Cr}$ atoms, we investigate all the $\left(2^{n-1}+1\right)$ spin configurations. For example, for $n=3$, we consider the nonmagnetic, FM ( $\uparrow \uparrow)$ and the three AFM states $(\downarrow \uparrow \uparrow, \uparrow \uparrow \downarrow$, and $\downarrow \uparrow \downarrow$ ) by setting different initial spin configurations on the $\mathrm{Cr}$ atoms. For substitutional doping of two and three $\mathrm{Cr}$ atoms, a complete search is conducted in the 72-atom cell, resulting in 21 (7 atomic configurations $\times 3$ spin states) and 105 (21 atomic configurations $\times 5$ spin states) fully relaxed calculations, respectively. The total energies clearly show that $\mathrm{Cr}$ ions prefer to get close to each other regardless of the magnetic states concerned. For substitutional doping of 4 (and 5) $\mathrm{Cr}$ atoms, we tested various configurations by adding the 
TABLE IV. Formation energies under metal-rich condition and spin moments for the different spin states of the structures involving direct $\mathrm{Cr}$-Cr bonding (see Fig. 3) as calculated in the 72-atom cell. The quantities in bold type are for the most favorable state. For structure I and II, the FM state is not stable.

\begin{tabular}{lccccc}
\hline \hline & Nonmagnetic & \multicolumn{2}{c}{ FM } & \multicolumn{2}{c}{ AFM } \\
\cline { 2 - 6 } Configuration & $\begin{array}{c}\text { Formation } \\
\text { energy }(\mathrm{eV} / \mathrm{Cr})\end{array}$ & $\begin{array}{c}\text { Formation } \\
\text { energy }(\mathrm{eV} / \mathrm{Cr})\end{array}$ & $\begin{array}{c}\text { Magnetic } \\
\text { moment }\left(\mu_{B} / \mathrm{Cr}\right)\end{array}$ & $\begin{array}{c}\text { Formation } \\
\text { energy }(\mathrm{eV} / \mathrm{Cr})\end{array}$ & $\begin{array}{c}\text { Magnetic } \\
\text { moment }\left(\mu_{B} / \mathrm{Cr}\right)\end{array}$ \\
\hline I & 6.672 & & & 6.634 & $1.35,-1.41$ \\
II & 6.892 & & & 6.791 & $0.66,-0.58$ \\
III & 5.176 & 4.757 & $3.45,3.45$ & $\mathbf{4 . 7 2 2}$ & $\mathbf{3 . 1 6},-\mathbf{3 . 1 6}$ \\
IV & 5.167 & 4.966 & $3.19,3.56$ & 4.843 & $2.99,-3.23$ \\
\hline \hline
\end{tabular}

extra $\mathrm{Cr}$ atom to the most favorable three (and four) Cr clusters in the cubic 96-atom cell.

Significantly, while the FM state is favored under pair doping, the AFM states of the two most favorable three$\mathrm{Cr}_{\mathrm{Ga}}$-doping configurations [Figs. 1(c) and 1(d)] are more favorable than the corresponding FM states, from calculations using both 72- and 96-atom cells. The calculated net spins of the two AFM states are $1.04 \mu_{B} / \mathrm{Cr}$ and $1.03 \mu_{B} / \mathrm{Cr}$, while the energy differences between the AFM and FM states are 72 and $132 \mathrm{meV}$ per 72-atom cell, and 113 and $243 \mathrm{meV}$ per 96-atom cell for the two structures, respectively. For higher concentrations of $\mathrm{Cr}_{\mathrm{Ga}}$, clustering still occurs with the AFM state favored. Moreover, the energy difference between the FM and the AFM states increases significantly with the cluster size and reaches $0.525 \mathrm{eV}$ (or $0.131 \mathrm{eV}$ per $\mathrm{Cr}$ ) for the 4- $\mathrm{Cr}_{\mathrm{Ga}}$ pyramid structure [Fig. 1(e)]. For the in-plane 4-Cr $\mathrm{Cr}_{\mathrm{Ga}}$ rhombus structure [Fig. 1(f)] with a net spin of $0.06 \mu_{B} / \mathrm{Cr}$, while the 96-atom cell shows that the AFM state is lower in energy by $12 \mathrm{meV}$ than the FM state, the 128atom cell predicts that the FM state is not stable, namely, the initial FM solution converges into an AFM state. For the 5-Cr $\mathrm{Ga}_{\mathrm{Ga}}$ bipyramid structure [Fig. 1(g)] with a net spin of $0.97 \mu_{B} / \mathrm{Cr}$, both the $96-, 108-$, and $256-$ atom cells show that the FM state is not stable. These results clearly demonstrate that as the size of the Cr clusters increases, AFM coupling becomes more and more favorable, as depicted in Fig. 4. This is in sharp contrast to the results for purely substitu-

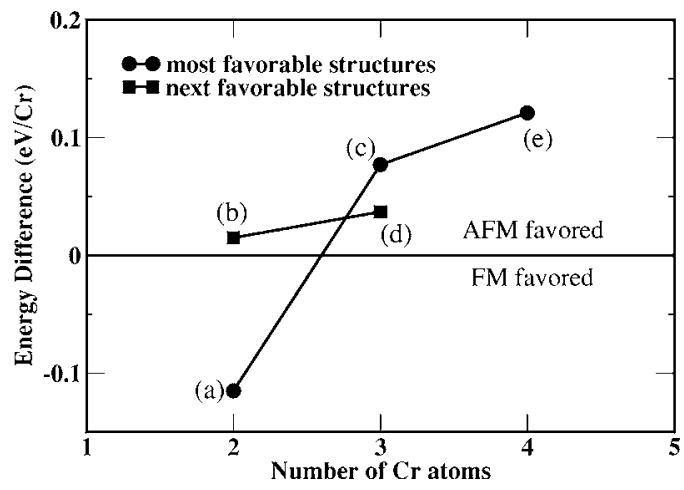

FIG. 4. Energy difference between the FM and AFM states per $\mathrm{Cr}$ atom for the lowest energy structures shown in Fig. 1. The letter labels correspond to the configurations in Fig. 1. For the structures in Fig. 1(f)-1(k), the FM states are predicted to be unstable. tional Mn:GaAs clusters where the FM alignment is always energetically favorable. ${ }^{47}$ We note, however, that Mahadevan and Zunger predicted that in Mn:GaAs the complex $\mathrm{Mn}_{\mathrm{Ga}}-\mathrm{Mn}_{\mathrm{i}}-\mathrm{Mn}_{\mathrm{Ga}}$ is actually more stable than pure $\mathrm{Mn}_{\mathrm{Ga}}$ clusters. ${ }^{45}$ Our results demonstrate that the stability and magnetic properties of small $\mathrm{Cr}$ clusters can be fundamentally altered by the presence of a third and more $\mathrm{Cr}$ atoms.

\section{E. Substitutional-interstitial complexes}

Since single interstitial doping of $\mathrm{Cr}$ is highly unfavorable, we considered various substitutional-interstitial complexes, with each containing only one octahedral interstitial $\mathrm{Cr}$ atom. For these configurations, we find that substitutional and interstitial dopants also prefer to occupy neighboring positions. All the favorable structures and spin configurations were refined in the large cubic 96-atom cell. Due to the large extent of the structures in Figs. 1(f) and 1(g), they were also calculated in the 128-atom cell for Fig. 1(f) and 108- and 256 atom cells for Fig. $1(\mathrm{~g})$.

For all the most favorable substitutional-interstitial complexes containing one $\mathrm{Cr}_{i-O}$ [Figs. 1(h)-1(k)], FM states are found unstable, i.e., self-consistent field calculations converge the initial FM configurations to AFM solutions, demonstrating the AFM interaction in these clusters. Interestingly, in all complexes containing more than $2-\mathrm{Cr}_{\mathrm{Ga}}$, there is a FM alignment between the $\mathrm{Cr}_{\mathrm{Ga}}$ atoms, while the magnetic interaction between the $\mathrm{Cr}_{i-O}$ and $\mathrm{Cr}_{\mathrm{Ga}}$ atoms is always antiparallel and the spin on $\mathrm{Cr}_{i \mathrm{o}}$ is considerably smaller. Note that in the complexes, the $\mathrm{Cr}_{i-O}$ and $\mathrm{Cr}_{\mathrm{Ga}}$ atoms are directly bonded, while the $\mathrm{Cr}_{\mathrm{Ga}}$ to $\mathrm{Cr}_{\mathrm{Ga}}$ atoms are bonded via $\mathrm{N}$ atoms. For all the structures considered, the nonmagnetic states are again found to be much higher in energy than the corresponding FM and AFM states.

Consistent with the total energies, one can also see the tendency for clustering by considering the average formation energy in Table II. The formation energies per $\mathrm{Cr}$ atom under different growth conditions decrease with increasing size of the $\mathrm{Cr}_{\mathrm{Ga}}$ cluster; that is, the $n-\mathrm{Cr}_{\mathrm{Ga}}$ clustering system is energetically more favorable than the $(n-1)-\mathrm{Cr}_{\mathrm{Ga}}$ system plus one isolated-Cr $\mathrm{Cr}_{\mathrm{Ga}}$ atom, showing that the embedded clusters tend to grow larger. From Table II, one can also see that for the substitutional configurations, the formation energies under N-rich condition are smaller than those under Ga-rich conditions. In contrast, the $\mathrm{Cr}_{\mathrm{N}}$, interstitial configurations, 
and the $\mathrm{Cr}-\mathrm{Cr}$ bonded structures are easier to achieve under Ga-rich growth conditions. Furthermore, it is clear that the formation energies for the substitutional-interstitial complexes are much higher than the same concentration pure substitutional configurations, showing that these configurations are rather unlikely, in agreement with recent experiments. ${ }^{12,24}$ This is strikingly different from the case of $\mathrm{Mn}$ :GaAs where the complex $\mathrm{Mn}_{\mathrm{Ga}}-\mathrm{Mn}_{\mathrm{i}}-\mathrm{Mn}_{\mathrm{Ga}}$ is more stable than are pure $\mathrm{Mn}_{\mathrm{Ga}}$ clusters. ${ }^{45}$ This difference may be attributed to the fact that GaAs is a "soft" semiconductor with a longer bond length and an interstitial free space of $3.7 \AA$ in diameter (excluding the ionic radii of neighboring ions) while GaN is a "hard" one and has an interstitial octahedral (tetrahedral) free space of $2.2 \AA$ (1.3 $)$ ), making interstitial site doping unfavorable.

\section{F. Local structural distortion and electronic properties}

As shown above, the energetically favored clusters consist of $\mathrm{Cr}$ atoms surrounding one central $\mathrm{N}$ atom. This is in line with the case of purely substitutional $\mathrm{Mn}$ clusters in Mn:GaAs. ${ }^{47}$ Regarding the atomic structure, an important feature is that the substitutional embedded clustering causes a strong local distortion, with the $\mathrm{Cr}-\mathrm{Cr}$ distances contracted by up to $11 \%$ [Fig. 1(e)] and $17 \%$ [Fig. 1(g)] as compared to the Ga-Ga distance in the host lattice-consistent with the strong attractive interaction between the $\mathrm{Cr}$ atoms. This can be contrasted to the case of a "single" $\mathrm{Cr}_{\mathrm{Ga}}$, where the $\mathrm{Cr}-\mathrm{N}$ bond is longer than the ideal Ga-N bond by $1.5 \%$ due to the larger $\mathrm{Cr}$ atomic radius $(1.40 \AA)$ than that of $\mathrm{Ga}(1.30 \AA)$. In the substitutional clusters, the contracted $\mathrm{Cr}-\mathrm{Cr}$ distances are due to the strong atomic attraction among the $\mathrm{Cr}$ atoms, regardless of the magnetic phases.

Interestingly, the experimental lattice constant of the $\mathrm{Cr}: \mathrm{GaN}$ system is found to systematically decrease with the $\mathrm{Cr}$ concentration more significantly in the $c$ axis than in the $a$ axis. ${ }^{12,21}$ This behavior can be understood by considering that the experimental samples are normally grown layer-bylayer along the [0001] direction; consequently, the embedded clusters are more readily grown along the $c$ axis than along the $a$ axis due to the attractive interaction between the dopants. The lattice constants are also found to decrease with increasing growth temperature up to the optimal temperature $T_{o}$ of $775^{\circ} \mathrm{C} .{ }^{12}$ This trend may be explained by the fact that the higher dopant mobility at elevated temperatures enhances the formation of $\mathrm{Cr}$ clusters. Above $T_{o}$, the fraction of substitutional $\mathrm{Cr}$ decreases sharply ${ }^{12}$ suggesting that this temperature is sufficient to form substitutional-interstitial complexes which result in an increase of the lattice parameter $c$-as we found for the most favorable structure with $\mathrm{Cr}_{i}$ [Fig. 1(k)] with the out-of-plane $\mathrm{Cr}-\mathrm{Cr}$ distance increased by $18 \%$ as compared to the Ga-Ga distance in the host lattice. This would result in the lattice parameter increasing if some $\mathrm{Cr}$ atoms occupy interstitial positions.

The strong local distortion also clearly shows that atomic relaxation is crucial for cluster-doped DMS systems. We note here that the geometries for different spin states vary only slightly, typically with $\mathrm{Cr}-\mathrm{Cr}$ distances changing by 0.01-0.05 A. Another important feature associated with the
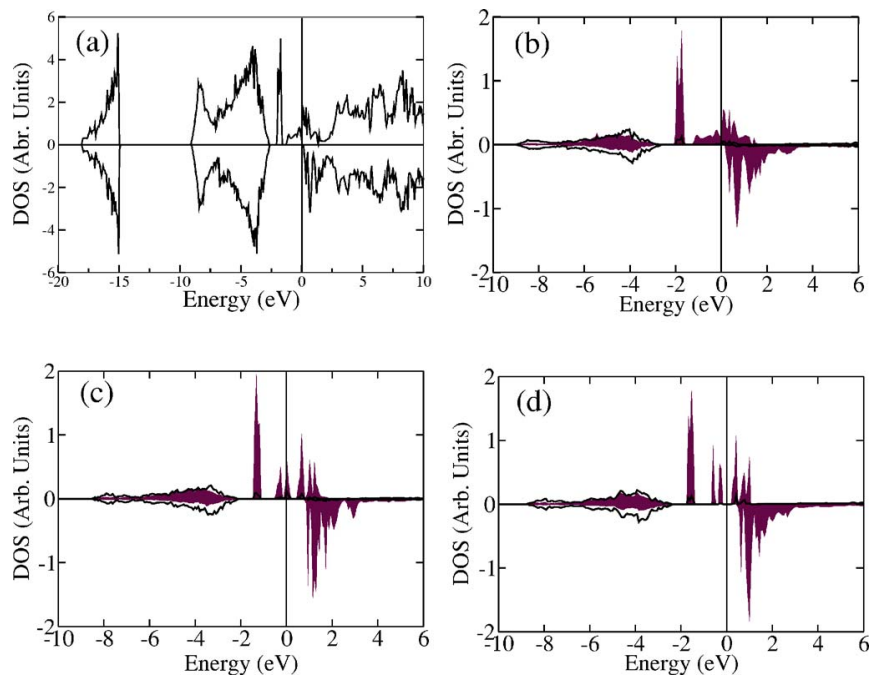

FIG. 5. (Color online) DOS for pair- $\mathrm{Cr}_{\mathrm{Ga}}$ [see Fig. 1(a)] in Cr:GaN: Total DOS (a) and the projected DOS (b) as calculated in the 32-atom cell. The projected DOS calculated using a 72-atom cell (c) and in a 96-atom cell (d). In (b), (c), and (d), a smaller energy range is shown as compared to (a). The filled grey areas are for $\mathrm{Cr}-3 d$ states and the solid lines are for the singly Cr-bonded $\mathrm{N}$ atom $2 p$ states.

clustering is the strong local polarization on the nearby $\mathrm{N}$ atoms (bonded to $\mathrm{Cr}$ ), for example, $0.15 \mu_{B}$ and $0.23 \mu_{B}$ are found for the structures shown in Figs. 1(c) and 1(e), respectively, compared to $0.05 \mu_{B}$ and $0.11 \mu_{B}$ on $\mathrm{N}$ for the case of isolated $\mathrm{Cr}_{\mathrm{Ga}}$. It is also interesting to note the nearly linear relationship between the atomic moment on the centered $\mathrm{N}$ atom with the coordination of $\mathrm{Cr}$ atoms. The large moments on the $\mathrm{N}$ atoms are due to hybridization between the $\mathrm{N}-2 p$ and $\mathrm{Cr}-3 d$ states. Hence, we propose that the "strong hybridization" found by soft x-ray emission and absorption spectroscopy measurements ${ }^{24}$ is due to the $\mathrm{N}-2 p$ and $\mathrm{Cr}-3 d$ states in embedded clustering configurations rather than the single

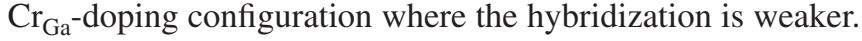

In addition to the fact that the dopant concentration affects the magnetic coupling, it is also important to observe the effect of $\mathrm{Cr}$ concentration on the electronic properties. DOS analysis reveals that isolated $\mathrm{Cr}_{\mathrm{Ga}}$ is $\mathrm{FM}$ and half metallic, in which the majority spin state is metallic and the minority spin state is insulating, in both the 32- and 72-atom cells (i.e., Cr concentrations of 6.25 and $2.78 \%$, respectively), which is consistent with FLAPW results. ${ }^{17}$ But in the 96atom cell (concentration of $2.08 \%$ ), it is a semiconductor, see Fig. 2. Similarly, for the most favorable pair-doping structure, Fig. 1(a), it is half metallic in the 32-atom cell, which is in agreement with previous theoretical results..22 However, both 72- and 96-atom cells predict a semiconducting behavior. The DOS plots for this configuration at different concentrations are compared in Fig. 5. For large cluster configurations, DOS calculations in the 96-atom cell show that structures depicted in Figs. 1(c) and 1(e) are both semiconductors while the higher concentration structure Fig. 1(g), is half metallic with numerous states in the band gap, see Fig. 6. This dependence of the electronic nature on supercell size can be understood in that the levels in the host band gap are 

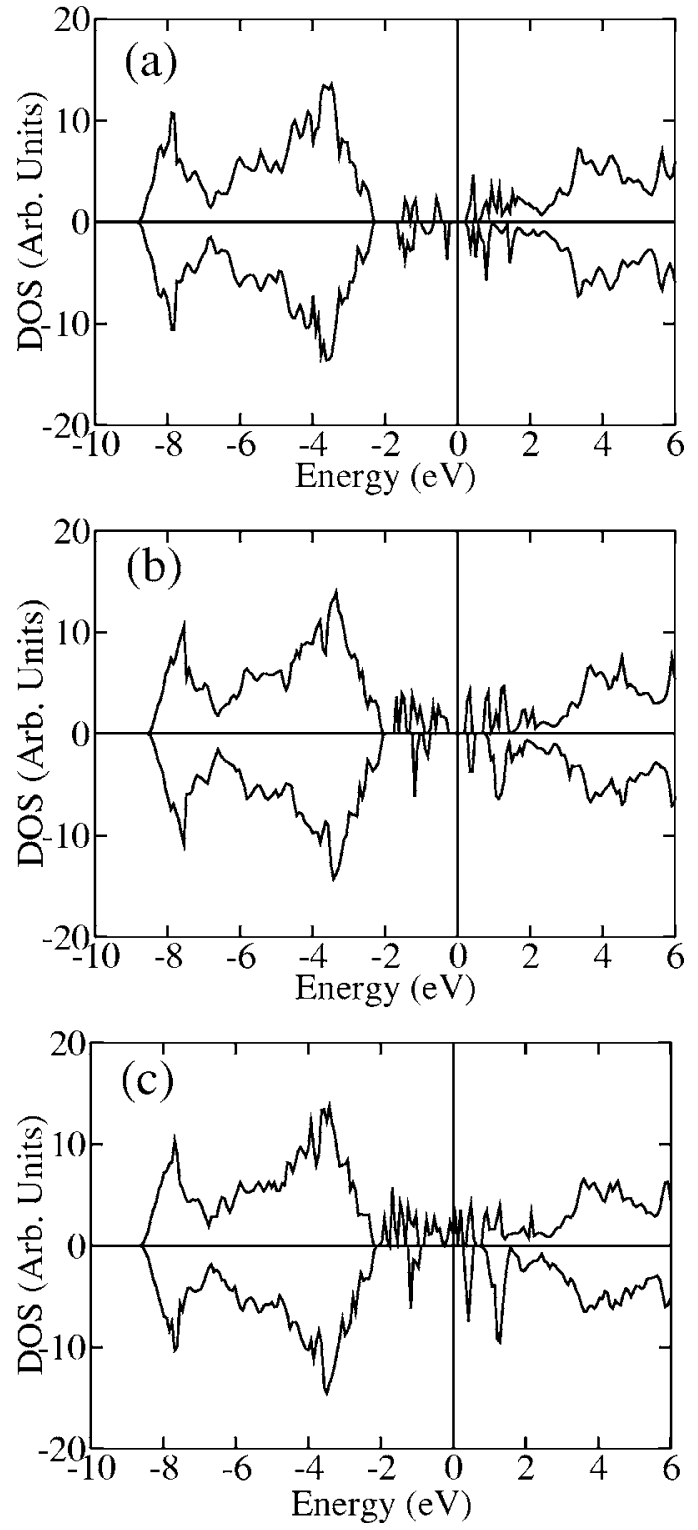

FIG. 6. Total DOS for cluster-doping configurations: (a) for Fig. 1(c), (b) for Fig. 1(e), and (c) for Fig. 1(g) as calculated in the cubic 96-atom cell.

derived from $\mathrm{Cr}-d$ states. Then, for high $\mathrm{Cr}$ concentrations (small cells) there will be significant interaction between the $\mathrm{Cr}$ atoms in neighboring cells, resulting in a broadening of the levels which may result in metallic or semimetallic behavior. In the large supercells these interactions become very weak giving rise to discrete levels, characteristic of "isolated defects." Interestingly, Lee et al. ${ }^{20}$ and Takeuchi et al. ${ }^{24}$ report that $\mathrm{Cr}: \mathrm{GaN}$ systems do exhibit semiconducting behavior. Thus, based on our theoretical calculations, we predict that the competition between metallic, half-metallic, and semiconducting behaviors depends sensitively on the dopant concentrations. Furthermore, there has been no consensus on whether the spin-spin interaction in DMS is long ranged or short ranged, as mentioned earlier. Our first-principles results clearly demonstrate that the interaction within the clusters is short ranged. However, the strong dependence of the magnetic coupling and electrical conductivity on supercell sizes indicates that the long-ranged interaction between the neighbouring clusters may also play a role in the electronic properties.

\section{DISCUSSION}

In order to validate the clustering of magnetic ions in DMS, it is important to consider the mobility of the dopants. In practice, since the equilibrium solubility of TMs is very low, III-V DMSs thin film samples have to be grown under nonequilibrium conditions. A nonequilibrium dynamical system is often thought as a complex energy landscape with many valleys and barriers. ${ }^{71}$ Mostly, molecular beam epitaxy (MBE) at low temperatures of the order of $250{ }^{\circ} \mathrm{C}$ has been used, but metal-organic vapor phase epitaxy at higher temperatures has also been employed. While not much is known quantitatively, $\mathrm{Cr}$ atoms are found to be mobile in GaN during sample growth using reactive MBE. ${ }^{72}$ Secondary ion mass spectroscopy has also confirmed diffusion of Mn atoms into GaN to a depth of $380 \AA{ }^{73}$ Furthermore, experiments on Mn:GaAs based digital heterostructures ${ }^{74}$ show that the substitutional ions can move several lattice-constants during growth at $230{ }^{\circ} \mathrm{C}$. Moreover, interstitial defects are believed to be much more mobile than the substitutional ones. ${ }^{18}$ These observations indicate that the doped magnetic ions are rather mobile at elevated temperatures and consequently clustering of the dopants in DMS is feasible. More directly, and in support of our findings, the clustering distribution of $\mathrm{Cr}$ in GaN films was clearly observed using energy-filtered imaging $^{31}$ and by analytical transmission electron microscopy on samples prepared below $775^{\circ} \mathrm{C} .{ }^{75}$ Moreover, structural characterization reveals that $\mathrm{Mn}: \mathrm{GaN}$ with $\mathrm{Mn}$ content of $13.7 \%$, nm-sized Mn-rich clusters are found to be embedded in the $(\mathrm{Ga}, \mathrm{Mn}) \mathrm{N}$ alloy and are coherent with the surrounding matrix. ${ }^{76}$

In Fig. 7, we present spin density contour plots of magnetic ground states for the single, pair [Fig. 1(a)] and trio [Fig. 1(c)] structures in the same (0001) plane obtained from the cubic 96 supercell calculations. In these states, while the single and pair configurations are both FM with the atomic moment close to $3 \mu_{B} / \mathrm{Cr}$, the adjacant $\mathrm{N}$ atoms are coupled antiferromagnetically with the $\mathrm{Cr}$ atom. However, the presence of the third $\mathrm{Cr}$ fundamentally alters such interactionsnow a state containing AFM coupling is the ground state. Such AFM states also apply for the larger clusters, as described earlier. Thus, our results clearly demonstrate that cluster-doping considerations are necessary to give a correct description of the magnetic coupling among the doped ions. The preference of AFM over FM for larger $\mathrm{Cr}$ clusters in $\mathrm{Cr}$ :GaN might not be surprising when one considers that AFM coupling is favored in both bulk $\mathrm{Cr}$ (Ref. 58) and gas phase $\mathrm{Cr}_{n}(n \geqslant 2)$ clusters, ${ }^{54}$ as well as in the CrN crystal. ${ }^{26,27}$

So far, we have concentrated on isolated cluster configurations; it will be important and interesting to investigate the interaction between the clusters. In this respect, we have calculated several cluster-cluster complexes and compared the total energy of the FM and AFM solutions. To do so, we put two previously investigated isolated clusters far apart in a large supercell and kept the initial spin structure within each 


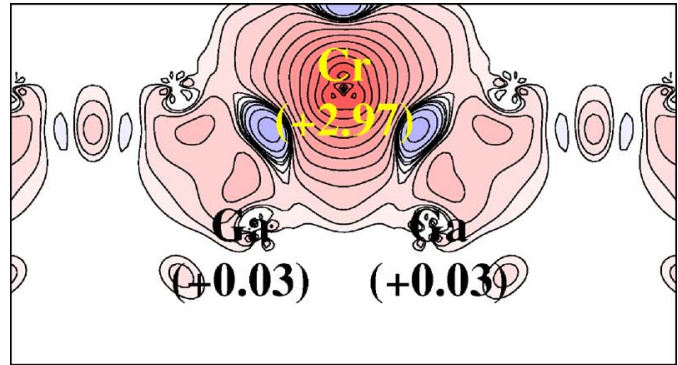

(a)

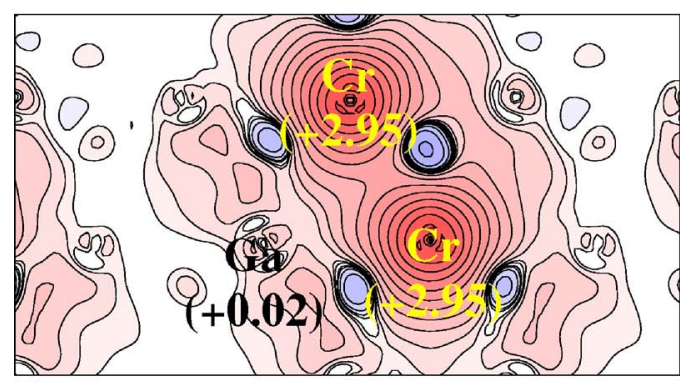

(b)

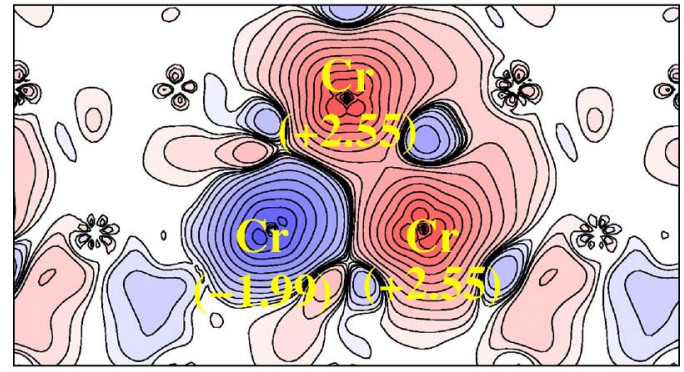

(c)

FIG. 7. (Color online) Spin density contour plots for the ground magnetic states of single (upper), pair (middle) [see Fig. 1(a)] and trio (lower) [see Fig. 1(c)] configurations in (0001) plane as calculated in the cubic 96-atom cell.

cluster as in the energetically favorable one. For each geometric configuration, we calculate two spin structures where the FM state is defined as majority-spin parallel and the AFM one as the majority-spin antiparallel. Specifically, for the single $\mathrm{Cr}_{\mathrm{Ga}}+$ pair $\mathrm{Cr}_{\mathrm{Ga}}$ [structure Fig. 1(a)] cluster interaction, using a $72 \times 2=144$ atom cell, FM is found favored over AFM by $12.5 \mathrm{meV} / \mathrm{cell}$; for a "pair+pair" cluster configuration, using a $72 \times 2=144$ atom cell, AFM is slightly favored over FM by $2.7 \mathrm{meV} / \mathrm{cell}$, while using a $96 \times 2$ $=192$ atom cell, FM is more stable by $4.7 \mathrm{meV} /$ cell. Using a $96 \times 2=192$ atom cell, we also calculated the "trio [structure Fig. 1(c)] + single" and "trio+pair" cluster configurations. Here the FM state is found more favorable than the AFM by 15.8 and $7.8 \mathrm{meV} / \mathrm{cell}$, respectively. We note that the energy differences between FM and AFM states are very small for all the cluster-cluster interaction configurations, suggesting a weak magnetic coupling between clusters. Furthermore, the energy differences and ground magnetic state are expected to be dependent on the distance and orientation, similar to the pair-doping results shown in Table III. This may give an atomic-scale explanation on the presence of the paramagnetic phase observed in experiments. ${ }^{21,81}$ In all these cases studied, the local magnetic structure within the clusters is almost unchanged due to the large distance between the clusters. From these considerations, we tend to conclude that the interaction between pair-cluster structures depends on the distance (maybe also orientation) between them, but for a dilute distribution of cluster configurations, FM coupling between pair clusters is predicted.

We believe that one of the main reasons for the lack of consensus regarding the origin of ferromagnetism in TMdoped DMS is due to the poor understanding so far of the atomic scale structure and associated magnetic properties, particularly the distribution of the magnetic ions. In addition to the attractive properties listed above, Cr-doped GaN systems are comparatively simple DMSs because (i) the secondary phase is rather unlikely, (ii) the clustering is found to be purely substitutional, and (iii) the $\mathrm{Cr}$ charge state is +3 in $\mathrm{Cr}: \mathrm{GaN},{ }^{21,70}$ and thus they may serve as the prototypical DMS system. Based on the present systematic total energy calculations and the mobility considerations, it is now possible to sketch a picture regarding the distribution and magnetism in Cr:GaN, and possibly other TM-doped DMS: The spatial distribution of the doped magnetic ions is not random or homogeneous, as frequently assumed in the literature. The $\mathrm{Cr}$ atoms prefer to form embedded clusters, substituting on cation sites, and maintaining the wurtzite structure. The size and geometries of these clusters will depend on a number of factors, e.g., the initial $\mathrm{Cr}$ positions, the extent of $\mathrm{Cr}-\mathrm{Cr}$ interactions, and the size of energy barriers for diffusion. Such an inhomogeneous distribution is in agreement with recent experiments by energy-filtered imaging ${ }^{31}$ and analytical transmission electron microscopy ${ }^{75}$ as recently demonstrated in $\mathrm{Cr}: \mathrm{GaN}$ and the theoretical work using Korringa-KohnRostoker coherent potential approximation method in Mn:GaN and Cr:ZnTe by Sato and co-workers. ${ }^{77,78}$ Various configurations with diverse magnetic structures will presumably coexist. Interestingly, from a long-range point of view, the experimentally observed "random distributed clusters" 31 will result in a "homogeneouslike" or "uniformlike" picture. For example, while both scanning Auger electron spectroscopy (AES) and secondary ion mass spectrometry (SIMS) profiling of the $\mathrm{Cr}$ atoms in AlN did not show any evidence of clustering within the limited resolution of these techniques (60 $\AA$ in the vertical direction for SIMS and, $100 \AA$ in the lateral resolution for the AES), ${ }^{56}$ evidence of $\mathrm{Cr}$ nanosegregation was determined by electron energy loss spectroscopy with a much higher resolution of $5 \AA$ probe size. ${ }^{7}$ The statistical distribution, and consequently the observed magnetism, will depend sensitively on details of the growth conditions. Indeed, cross-sectional scanning tunneling microscopy (STM) of Mn-doped GaAs (110) surfaces identify that three types of Mn configurations (single-Mn, pair substitutional $\mathrm{Mn}_{\mathrm{Ga}}$ configurations, and substitutional-interstitial complexes) coexist on the surface. ${ }^{79}$ Moreover, recent x-ray scanning microscopy directly observed that in $\mathrm{Mn}: \mathrm{GaN}$, the $\mathrm{Mn}$ atoms are "somewhat inter-related among themselves" and that they are clearly not randomly distributed and the Mn elemental map showed "Mn cluster" formation on the micrometer scale. ${ }^{80}$ In addition, a weak AFM coupling between the implanted ions was observed in $n$ - and $p$-type GaN layers implanted with $\mathrm{Mn}, \mathrm{Cr}$, and $\mathrm{V} .{ }^{81}$ Although more evidence is 
required, we propose that this picture may apply to other DMS systems. Indeed, we find a very similar behavior for $\mathrm{Cr}: \mathrm{AlN}, \mathrm{Mn}: \mathrm{AlN}$, and Mn:GaN. ${ }^{82}$ Also, for the consideration of magnetic-atom pair doping, first-principles calculations for $\mathrm{Co}: \mathrm{GaN}$ (Ref. 83) and $\mathrm{Co}: \mathrm{TiO}_{2}$ (Ref. 84) show that the dopant atoms prefer to occupy neighboring cation sites. Furthermore, recent experimental results clearly established the presence of nanosized superparamagnetic cobalt "metal clusters" of $8-10 \mathrm{~nm}$ size in the film $\mathrm{Co}: \mathrm{TiO}_{2}$, located at the film-substrate interface. ${ }^{85}$

With this picture, one can further understand some experimental observations. (i) The predicted existence of AFM clusters (not necessarily restricted to the configurations studied here with net spins ranging between $0.06-1.47 \mu_{B} / \mathrm{Cr}$ as larger clusters may also appear) is believed to contribute to the low mean magnetic moment $\left(0.45-1.1 \mu_{B} / \mathrm{Cr}\right)$ observed in experiments ${ }^{10,12,20}$ and possibly contribute to the anomalous fluctuation detected in magnetization curves. ${ }^{18}$ (ii) With increasing $\mathrm{Cr}$ concentration it has been found experimentally that the mean magnetic moment is strongly suppressed for both Cr:GaN (Ref. 21) and Cr:AlN. ${ }^{86}$ This can be naturally understood in that as the concentration is increased, the $\mathrm{Cr}$ atoms increasingly experience an attractive interaction and form AFM clusters which results in a lower net magnetization, as intuitively expected by Kim et al. ${ }^{21}$ For Mn:GaAs, while STM experiments do not show signs of clustering with a low doping concentration of $0.5 \%,{ }^{87}$ with a concentration of $1 \%$, substitutional clustering formation was clearly demonstrated. ${ }^{79}$ (iii) Our picture also helps one to understand the role of annealing treatment in relation to the magnetic properties of DMS: A gradual decay in the spin moment of Cr-doped GaN has been observed, from 1.8 to $0.45 \mu_{B} / \mathrm{Cr}$, over a period of annealing time. ${ }^{12}$ Annealing at $250{ }^{\circ} \mathrm{C}$ for longer than $2 \mathrm{~h}$ in Mn:GaAs leads to a reduction in its ferromagnetism. ${ }^{88}$ Microscopically, the annealing can be regarded as a redistribution process for the magnetic ions, and this rearrangement significantly enhances the formation of AFM clusters, resulting in a change in the observed magnetization.

\section{CONCLUSIONS}

We presented a comprehensive poly-doping investigation on $\mathrm{Cr}: \mathrm{GaN}$, based on first-principles density functional theory. Our systematic calculations show that the distribution of $\mathrm{Cr}$ ions in $\mathrm{GaN}$ is neither homogenous nor random, as widely assumed. Rather, under both $\mathrm{Ga}$ - and N-rich growth conditions, the doped $\mathrm{Cr}$ atoms prefer to occupy Ga sites and have a strong tendency to form embedded clusters. Substitutional configurations are more favorable than substitutionalinterstitial complexes. For $\mathrm{Cr}$ clusters larger than two atoms, states containing AFM coupling are energetically preferred over FM and nonmagnetic states. We propose a picture that various cluster configurations coexist and the statistical distribution and consequently the magnetism depends sensitively on the growth conditions. These findings elucidate various hitherto puzzling experimental results-such as (i) much lower value of the measured saturation magnetic moment on $\mathrm{Cr}$ as compared to the theoretically predicted one for the isolated dopants, (ii) variation in the lattice constant with concentration and temperature, and (iii) the observed strong dependence of the magnetization on the $\mathrm{Cr}$ concentration, growth temperature, and annealing. Hence this work represents progress towards understanding the intrinsic complex nature of the distribution and magnetism in TM-doped DMS. We also emphasize that the dopant concentration is crucial to predict the magnetic and electronic properties. We find very similar results for $\mathrm{Mn}$ in $\mathrm{GaN}$ and $\mathrm{Cr}$ and $\mathrm{Mn}$ in AlN and argue such a scenario may also hold for other dilute magnetic semiconductor systems.

\section{ACKNOWLEDGMENTS}

We acknowledge the computing resources provided by the Australian Partnership for Advanced Computing National Facility and by the Australian Centre for Advanced Computing and Communications and the financial support by the Australian Research Council. Work at Northwestern University was supported by the NSF (through its MRSEC program).
${ }^{1}$ H. Munekata, H. Ohno, S. von Molnar, A. Segmuller, L. L. Chang, and L. Esaki, Phys. Rev. Lett. 63, 1849 (1989).

${ }^{2}$ H. Ohno, A. Shen, F. Matsukura, A. Oiwa, A. Endo, S. Katsumoto, and Y. Iye, Appl. Phys. Lett. 69, 363 (1996); H. Ohno, Science 281, 951 (1998).

${ }^{3}$ S. A. Wolf, D. D. Awschalom, R. A. Buhrman, J. M. Daughton, S. von Molnar, M. L. Roukes, A. Y. Chtchelkanova, and D. M. Treger, Science 294, 1488 (2001); I. Zutic, J. Fabian, and S. D. Sarma, Rev. Mod. Phys. 76, 323 (2004).

${ }^{4}$ Spin Electronics, edited by M. Ziese and M. J. Thornton (Springer, Berlin, 2001).

${ }^{5}$ B. Beschoten, E. Johnston-Halperin, D. K. Young, M. Poggio, J. E. Grimaldi, S. Keller, S. P. DenBaars, U. K. Mishra, E. L. Hu, and D. D. Awschalom, Phys. Rev. B 63, 121202(R) (2001).

${ }^{6}$ S. Krishnamurthy, M. van Schilfgaarde, and N. Newman, Appl.
Phys. Lett. 83, 1761 (2003).

${ }^{7}$ S. Y. Wu, H. X. Liu, L. Gu, R. K. Singh, L. Budd, M. van Schilfgaarde, M. R. McCartney, D. J. Smith, and N. Newman, Appl. Phys. Lett. 82, 3047 (2003).

${ }^{8}$ T. Dietl, H. Ohno, F. Matsukura, J. Cibert, and D. Ferrand, Science 287, 1019 (2000).

${ }^{9}$ M. Hashimoto, Y. K. Zhou, M. Kanamura, and H. Asahi, Solid State Commun. 122, 37 (2002).

${ }^{10}$ S. E. Park, H. J. Lee, Y. C. Cho, S. Y. Jeong, C. R. Cho, and S. Cho, Appl. Phys. Lett. 80, 4187 (2002).

${ }^{11}$ H. X. Liu, S. Y. Wu, R. K. Singh, L. Gu, D. J. Smith, N. Newman, N. R. Dilley, L. Montes, and M. B. Simmonds, Appl. Phys. Lett. 85, 4076 (2004).

${ }^{12}$ R. K. Singh, S. Y. Wu, H. X. Liu, L. Gu, D. J. Smith, and N. Newman, Appl. Phys. Lett. 86, 012504 (2005). 
${ }^{13}$ A. Y. Polyakov, N. B. Smirnov, A. V. Govorkov, S. J. Pearton, and J. M. Zavada, J. Phys.: Condens. Matter 16, 2967 (2004).

${ }^{14}$ T. Graf, S. T. B. Goennenwein, and M. S. Brandt, Phys. Status Solidi B 239, 277 (2003).

${ }^{15}$ Y. K. Zhou, M. Hashimoto1, M. Kanamura, and H. Asahil, J. Supercond. 16, 37 (2003).

${ }^{16}$ K. Sato, P. H. Dederics, and H. Katayama-Yoshida, Europhys. Lett. 61, 403 (2003).

${ }^{17}$ J. E. Medvedeva, A. J. Freeman, X. Y. Cui, C. Stampfl, and N. Newman, Phys. Rev. Lett. 94, 146602 (2005).

${ }^{18}$ S. J. Pearton, C. R. Abernathy, M. E. Overberg, G. T. Thaler, D. P. Norton, N. Theodoropoulou, A. F. Hebard, Y. D. Park, F. Ren, J. Kim, and L. A. Boatner, J. Appl. Phys. 93, 1 (2003); C. Timm, J. Phys.: Condens. Matter 15, R1865 (2003).

${ }^{19}$ S. A. Chambers, Surf. Sci. Rep. 61, 345 (2006).

${ }^{20}$ J. S. Lee, J. D. Lim, Z. G. Khim, Y. D. Park, S. J. Pearton, and S. N. G. Chu, J. Appl. Phys. 93, 4512 (2003).

${ }^{21}$ J. J. Kim, H. Makino, M. Sakurai, D. C. Oh, T. Hanada, M. W. Cho, T. Yao, S. Emura, and K. Kobayashi, J. Vac. Sci. Technol. B 23, 1308 (2005).

${ }^{22}$ G. P. Das, B. K. Rao, and P. Jena, Phys. Rev. B 69, 214422 (2004).

${ }^{23}$ N. Tandon, G. P. Das, and A. Kshirsagar, J. Phys.: Condens. Matter 18, 9245 (2006).

${ }^{24}$ T. Takeuchi, Y. Harada, T. Tokushima, M. Taguchi, Y. Takata, A. Chainani, J. J. Kim, H. Makino, T. Yao, T. Yamamoto, T. Tsukamoto, S. Shin, and K. Kobayashi, Phys. Rev. B 70, 245323 (2004).

${ }^{25}$ K. Yamaguchi, H. Tomioka, T. Yui, T. Suemasu, K. Ando, R. Yoshizaki, and F. Hasegawa, Jpn. J. Appl. Phys., Part 1 44, 6510 (2005).

${ }^{26}$ L. M. Corliss, N. Elliott, and J. M. Hastings, Phys. Rev. 117, 929 (1960).

${ }^{27}$ K. Suzuki, T. Kaneko, H. Yoshida, Y. Obi, and H. Fujimori, J. Alloys Compd. 280, 294 (1998).

${ }^{28}$ U. Haussermann, P. Viklund, M. Bostrom, R. Norrestam, and S. I. Simak, Phys. Rev. B 63, 125118 (2001).

${ }^{29}$ J. D. Browne, P. R. Liddell, R. Street, and T. Mills, Phys. Status Solidi A 1, 715 (1970).

${ }^{30}$ S. G. Yang, A. B. Pakhomov, S. T. Hung, and C. Y. Wong, Appl. Phys. Lett. 81, 2148 (2002).

${ }^{31}$ L. Gu, S. Y. Wu, H. X. Liu, R. K. Singh, N. Newman, and D. J. Smith, J. Magn. Magn. Mater. 290-291, 1395 (2005).

${ }^{32}$ M. Berciu and R. N. Bhatt, Phys. Rev. Lett. 87, 107203 (2001).

${ }^{33}$ C. Timm, F. Schafer, and F. von Oppen, Phys. Rev. Lett. 89, 137201 (2002).

${ }^{34}$ J. L. Xu, M. van Schilfgaarde, and G. D. Samolyuk, Phys. Rev. Lett. 94, 097201 (2005).

${ }^{35}$ L. Bergqvist, O. Eriksson, J. Kudrnovsky, V. Drchal, P. Korzhavyi, and I. Turek, Phys. Rev. Lett. 93, 137202 (2004).

${ }^{36}$ K. Sato, W. Schweika, P. H. Dederichs, and H. KatayamaYoshida, Phys. Rev. B 70, 201202(R) (2004).

${ }^{37}$ G. Bouzerar, T. Ziman, and J. Kudrnovsky, Europhys. Lett. 69, 812 (2005).

${ }^{38}$ K. Sato and H. Katayama-Yoshida, Jpn. J. Appl. Phys., Part 2 40, L485 (2001); Y.-J. Zhao, W. T. Geng, K. T. Park, and A. J. Freeman, Phys. Rev. B 64, 035207 (2001); L. Kronik, M. Jain, and J. R. Chelikowsky, ibid. 66, 041203 (2002); E. Kulatov, H. Nakayama, H. Mariette, H. Ohta, and Y. A. Uspenskii, ibid. 66, 045203 (2002); B. Sanyal, O. Bengone, and S. Mirbt, ibid. 68,
205210 (2003); S. H. Wei, X. G. Gong, G. M. Dalpian, and S. H. Wei, ibid. 71, 144409 (2005).

${ }^{39}$ Y. J. Zhao, T. Shishidou, and A. J. Freeman, Phys. Rev. Lett. 90, 047204 (2003).

${ }^{40}$ Q. Wang, Q. Sun, P. Jena, J. Z. Yu, R. Note, and Y. Kawazoe, Phys. Rev. B 72, 045435 (2005).

${ }^{41}$ P. Mahadevan, J. M. Osorio-Guillen, and A. Zunger, Appl. Phys. Lett. 86, 172504 (2005).

${ }^{42}$ Q. Wang, A. K. Kandalam, Q. Sun, and P. Jena, Phys. Rev. B 73, 115411 (2006).

${ }^{43}$ G. P. Das, B. K. Rao, and P. Jena, Phys. Rev. B 68, 035207 (2003).

${ }^{44}$ M. van Schilfgaarde and O. N. Mryasov, Phys. Rev. B 63, 233205 (2001).

${ }^{45}$ P. Mahadevan and A. Zunger, Phys. Rev. B 68, 075202 (2003).

${ }^{46}$ V. Drchaly, J. Kudrnovsky, I. Turek, F. Maca, and P. Weinberger, Philos. Mag. 84, 1889 (2004).

${ }^{47}$ H. Raebiger, A. Ayuela, and J. von Boehm, Phys. Rev. B 72, 014465 (2005).

${ }^{48}$ L. M. Sandratskii, P. Bruno, and S. Mirbt, Phys. Rev. B 71, 045210 (2005).

${ }^{49}$ B. Delley, J. Chem. Phys. 113, 7756 (2000); 92, 508 (1990).

${ }^{50}$ J. P. Perdew, K. Burke, and M. Ernzerhof, Phys. Rev. Lett. 77, 3865 (1996).

${ }^{51}$ B. Delley, Phys. Rev. B 66, 155125 (2002).

${ }^{52}$ C. Stampfl and C. G. Van de Walle, Phys. Rev. B 59, 5521 (1999), and references therein.

${ }^{53}$ H. J. Monkhorst and J. D. Pack, Phys. Rev. B 13, 5188 (1976).

${ }^{54}$ H. S. Cheng and L. S. Wang, Phys. Rev. Lett. 77, 51 (1996).

${ }^{55}$ S. Picozzi, T. Shishidou, A. J. Freeman, and B. Delley, Phys. Rev. B 67, 165203 (2003).

${ }^{56}$ R. M. Frazier, G. T. Thaler, J. Y. Leifer, J. K. Hite, B. P. Gila, C. R. Abernathy, and S. J. Pearton, Appl. Phys. Lett. 86, 052101 (2005).

${ }^{57}$ A. Y. Polyakov, N. B. Smirnov, A. V. Govorkov, R. M. Frazier, J. Y. Leifer, G. T. Thaler, C. R. Abernathy, S. J. Pearton, and J. M. Zavada, Appl. Phys. Lett. 85, 4067 (2004).

${ }^{58}$ R. Hafner, D. Spisak, R. Lorenz, and J. Hafner, Phys. Rev. B 65, 184432 (2002).

${ }^{59}$ C. G. Van de Walle, J. Appl. Phys. 95, 3851 (2004).

${ }^{60}$ K. Hirai, J. Phys. Soc. Jpn. 66, 560 (1997).

${ }^{61}$ J. M. Simmons, Metall. Mater. Trans. A 26, 2579 (1995).

${ }^{62}$ Handbook of Chemistry and Physics, edited by R. C. Weast, M. J. Astle, and W. H. Beyer (Chemical Rubber Co., Boca Raton, FL, 1988), Vol. 62.

${ }^{63}$ V. L. Moruzzi, Phys. Rev. Lett. 57, 2211 (1986).

${ }^{64}$ W. E. Pickett, Comput. Phys. Rep. 9, 115 (1989).

${ }^{65}$ C. Kittel, Introduction to Solid State Physics (New York, Wiley, 1986).

${ }^{66}$ B. D. Sharma and J. Donohue, Z. Kristallogr. Z. Kristallogr. 117, 293 (1962).

${ }^{67}$ P. A. Korzhavyi, I. A. Abrikosov, E. A. Smirnova, L. Bergqvist, P. Mohn, R. Mathieu, P. Svedlindh, J. Sadowski, E. I. Isaev, Yu. Kh. Vekilov, and O. Eriksson, Phys. Rev. Lett. 88, 187202 (2002).

${ }^{68}$ J. Neugebauer and C. G. Van de Walle, Phys. Rev. B 50, 8067 (1994).

${ }^{69}$ S. Limpijumnong and C. G. Van de Walle, Phys. Rev. B 69, 035207 (2004).

${ }^{70}$ S. Subashchandran, S. Kimura, M. S. Kim, S. Kobayashi, Y. K. 
Zhou, S. Hasegawa, and H. Asahi, Jpn. J. Appl. Phys., Part 2 45, 3522 (2006).

${ }^{71}$ M. Goldstein, J. Chem. Phys. 51, 3728 (1969).

${ }^{72}$ N. Newman (private communication).

${ }^{73}$ M. L. Reed, M. K. Ritums, H. H. Stadelmaier, M. J. Reed, C. A. Parker, S. M. Bedair, and N. A. El-Masry, Mater. Lett. 51, 500 (2001).

${ }^{74}$ R. K. Kawakami, E. Johnston-Halperin, L. F. Chen, M. Hanson, N. Guebels, J. S. Speck, A. C. Gossard, and D. D. Awschalom, Appl. Phys. Lett. 77, 2379 (2000); E. Johnston-Halperin, J. A. Schuller, C. S. Gallinat, T. C. Kreutz, R. C. Myers, R. K. Kawakami, H. Knotz, A. C. Gossard, and D. D. Awschalom, Phys. Rev. B 68, 165328 (2003).

${ }^{75}$ S. Y. Wu and N. Newman, Appl. Phys. Lett. 89, 142105 (2006).

${ }^{76}$ S. Dhar, O. Brandt, A. Trampert, L. Daweritz, K. J. Friedland, K. H. Ploog, J. Keller, B. Beschoten, and G. Guntherodt, Appl. Phys. Lett. 82, 2077 (2003).

${ }^{77}$ K. Sato, H. Katayama-Yoshida, and P. H. Dederichs, Jpn. J. Appl. Phys., Part 2 44, L948 (2005).

${ }^{78}$ T. Fukushima, K. Sato, H. Katayama-Yoshida, and P. H. Dederichs, Jpn. J. Appl. Phys., Part 2 45, L416 (2006).
${ }^{79}$ J. M. Sullivan, G. I. Boishin, L. J. Whitman, A. T. Hanbicki, B. T. Jonker, and S. C. Erwin, Phys. Rev. B 68, 235324 (2003).

${ }^{80}$ G. Martinez-Criado, A. Somogyi, M. Hermann, M. Eickhoff, and M. Stutzmann, Jpn. J. Appl. Phys., Part 2 43, L695 (2004).

${ }^{81}$ V. A. Guzenko, N. Thillosen, A. Dahmen, R. Calarco, Th. Schapers, L. Houben, M. Luysberg, B. Schineller, M. Heuken, and A. Kaluza, J. Appl. Phys. 96, 5663 (2004).

${ }^{82}$ X. Y. Cui, B. Delley, A. J. Freeman, and C. Stampfl (unpublished).

${ }^{83}$ J. S. Hong and R. Q. Wu, Phys. Rev. B 68, 233306 (2003).

${ }^{84}$ Z. X. Yang, G. Liu, and R. Q. Wu, Phys. Rev. B 67, 060402(R) (2003).

${ }^{85}$ S. R. Shinde, S. B. Ogale, J. S. Higgins, H. Zheng, A. J. Millis, V. N. Kulkarni, R. Ramesh, R. L. Greene, and T. Venkatesan, Phys. Rev. Lett. 92, 166601 (2004).

${ }^{86}$ D. Kumar, J. Antifakos, M. G. Blamire, and Z. H. Barber, Appl. Phys. Lett. 84, 5004 (2004).

${ }^{87}$ B. Grandidier, J. P. Nys, C. Delerue, D. Stiévenard, Y. Higo, and M. Tanaka, Appl. Phys. Lett. 68, 235324 (2000).

${ }^{88}$ S. J. Potashnik, K. C. Ku, S. H. Chun, J. J. Berry, N. Samarth, and P. Schiffer, Appl. Phys. Lett. 79, 1495 (2001). 